\title{
Multi-Dimensional Extensions of the Chebyshev Polynomials
}

\author{
By Richard O. Hays
}

\begin{abstract}
Two families of polynomials are introduced which satisfy multi-dimensional (or multi-indiced) recursion relationships. These polynomials are developed from the Chebyshev polynomials. Also two additional polynomials are presented which satisfy a special twodimensional recursion relationship.
\end{abstract}

I. Introduction. The Chebyshev polynomials belong to the set of ultraspherical or Gegenbauer polynomials and are related to the hypergeometric functions [1]. These polynomials have proven useful in such areas as lattice dynamics [2], numerical analysis [1], and differential equations [1], [3].

The Chebyshev polynomials appear in the literature in various forms, so the following relationships define the forms of the polynomials which will be employed herein [1]:

$$
\begin{aligned}
& \frac{1-x^{2}}{1-2 \alpha x+x^{2}}=T(0 ; \alpha)+2 \sum_{n=1}^{\infty} T(n ; \alpha) x^{n}, \\
& \frac{1}{1-2 \alpha x+x^{2}}=\sum_{n=0}^{\infty} U(n ; \alpha) x^{n},
\end{aligned}
$$

where $T(n ; \alpha)$ and $U(n ; \alpha)$ are the Chebyshev polynomials of the first and second kind, respectively, and $T(0 ; \alpha) \equiv 1$.

The terms $\left(1-x^{2}\right) /\left(1-2 \alpha x+x^{2}\right)$ and $1 /\left(1-2 \alpha x+x^{2}\right)$ are the generating functions for the Chebyshev polynomials of the first and second kind, respectively, where the expressions (1.1) and (1.2) are valid, provided $|x|<\min \left|\alpha \pm\left(\alpha^{2}-1\right)^{1 / 2}\right|$.

The expressions for $T(n ; \alpha)$ and $U(n ; \alpha)$ are

$$
\begin{aligned}
& T(n ; \alpha)=\frac{n}{2} \sum_{m=0}^{|n / 2|} \frac{(-)^{m}(n-m-1) !}{m !(n-2 m) !}(2 \alpha)^{n-2 m}, \\
& U(n ; \alpha)=\sum_{m=0}^{|n / 2|} \frac{(-)^{m}(n-m) !}{m !(n-2 m) !}(2 \alpha)^{n-2 m} .
\end{aligned}
$$

Let $I(n ; \alpha)$ represent either $T(n ; \alpha)$ or $U(n ; \alpha)$; then $I(n ; \alpha)$ satisfies the recursion relationship

$$
2 \alpha I(n+1 ; \alpha)-I(n+2 ; \alpha)-I(n ; \alpha)=0 .
$$

II. Extensions to Two Dimensions. The Chebyshev polynomials can be extended to two dimensions by forming multivariate generating functions produced

Received May 22, 1972.

AMS (MOS) subject classifications (1970). Primary 05A15, 32A05, 33A65, 39A10.

Copyright $\subseteq 1973$, American Mathematical Society 
by replacing $\alpha$ by $\left(\alpha-\left(y+y^{-1}\right) / 2\right)$ in the original generating functions. Employing the multinomial theorem, we find that

$$
\begin{aligned}
& T\left(n ; \alpha-\left(y+y^{-1}\right) / 2\right)=\sum_{r=-n}^{n} T(n ; r ; \alpha) y^{r}, \\
& U\left(n ; \alpha-\left(y+y^{-1}\right) / 2\right)=\sum_{r=-n}^{n} U(n ; r ; \alpha) y^{r},
\end{aligned}
$$

where

$$
\begin{aligned}
& T(n ; r ; \alpha)=\frac{n}{2} \sum_{m=0}^{[n / 2]} \frac{(-)^{m+r}(n-m-1) !}{m !} \sum_{k=0}^{[\beta \mid} \frac{K(2 \alpha)^{q} H(q)}{q !}, \\
& U(n ; r ; \alpha)=\sum_{m=0}^{\mid n / 2]} \frac{(-)^{m+r}(n-m) !}{m !} \sum_{k=0}^{[\beta]} \frac{K(2 \alpha)^{a} H(q)}{q !}
\end{aligned}
$$

subject to the relations

$$
\begin{aligned}
\beta & =(n-|r|-2 m) / 2, \\
K & =1 / k !(k+|r|) !, \\
q & =n-|r|-2 m-2 k,
\end{aligned}
$$

and where $H(q)$ is the Heaviside step function,

$$
H(q)=\left\{\begin{array}{ll}
0 & \text { if } q<0 \\
1 & \text { if } q \geqq 0
\end{array}\right\} .
$$

$I(n ; r ; \alpha)$ satisfies the recursion relationship

$$
\begin{aligned}
2 \alpha I(n+1 ; r+1 ; \alpha) & -I(n+2 ; r+1 ; \alpha)-I(n ; r+1 ; \alpha) \\
& -I(n+1 ; r+2 ; \alpha)-I(n+1 ; r ; \alpha)=0,
\end{aligned}
$$

where $I(n ; r ; \alpha)$ represents either $T(n ; r ; \alpha)$ or $U(n ; r ; \alpha)$.

Several of the $U(n ; r ; \alpha)$ polynomials are displayed in Table I.

TABLE I

$U(n ; r ; \alpha)$ Polynomials

\begin{tabular}{l|lllllll}
\hline & \multicolumn{7}{|c}{$r$} \\
\cline { 2 - 7 }$n$ & 0 & 1 & 2 & 3 & 4 & 5 \\
\hline 0 & 1 & 0 & 0 & 0 & 0 & 0 \\
1 & $2 \alpha$ & -1 & 0 & 0 & 0 & 0 \\
2 & $4 \alpha^{2}+1$ & $-4 \alpha$ & 1 & 0 & 0 & 0 \\
3 & $8 \alpha^{3}+8 \alpha$ & $-12 \alpha^{2}-1$ & $6 \alpha$ & -1 & 0 & 0 \\
4 & $16 \alpha^{4}+36 \alpha^{2}+1$ & $-32 \alpha^{3}-12 \alpha$ & $24 \alpha^{2}+1$ & $-8 \alpha$ & 1 & 0 \\
5 & $32 \alpha^{6}+128 \alpha^{3}+18 \alpha$ & $-80 \alpha^{4}-72 \alpha^{2}-1$ & $80 \alpha^{3}+16 \alpha$ & $-40 \alpha^{2}-1$ & $10 \alpha$ & -1 \\
\hline
\end{tabular}


III. Extensions to $N+1$ Dimensions. The generalization to $N+1$ dimensions is straightforward with the replacement of $\alpha$ by

$$
\left(\alpha-\frac{y_{1}+y_{1}^{-1}+y_{2}+y_{2}^{-1}+\cdots+y_{N}+y_{N}^{-1}}{2}\right)
$$

in the generating functions for the original Chebyshev polynomials.

$T$ and $U$ are given by

$$
\begin{aligned}
& T\left(n ; r_{1}, r_{2}, \cdots, r_{N} ; \alpha\right) \\
& \quad=\frac{n}{2} \sum_{m=0}^{[n / 2]} \frac{(-)^{m+\gamma}(n-m-1) !}{m !} \sum_{k_{1}=0}^{\left[\beta_{1}\right]} K_{1} \sum_{k_{2}=0}^{\left[\beta_{2}\right]} K_{2} \cdots \sum_{k_{N}=0}^{\left[\beta_{N}\right]} \frac{K_{N}(2 \alpha)^{q} H(q)}{q !}, \\
& U\left(n ; r_{1}, r_{2}, \cdots, r_{N} ; \alpha\right) \\
& \quad=\sum_{m=0}^{[n / 2]} \frac{(-)^{m+\gamma}(n-m) !}{m !} \sum_{k_{1}=0}^{\left[\beta_{1}\right]} K_{1} \sum_{k_{2}=0}^{\left[\beta_{2}\right]} K_{2} \cdots \sum_{K_{N}=0}^{\left[\beta_{N}\right]} \frac{K_{N}(2 \alpha)^{q} H(q)}{q !}
\end{aligned}
$$

with

$$
\begin{aligned}
& \gamma=r_{1}+r_{2}+\cdots+r_{N}, \\
& \beta_{p}=\left(n-\left|r_{1}\right|-\left|r_{2}\right|-\cdots-\left|r_{p}\right|-2 m-2 k_{1}-2 k_{2}-\cdots-2 k_{p-1}\right) / 2
\end{aligned}
$$

for $p=1,2,3, \cdots, N$, if we define $k_{0}=0$,

$$
\begin{aligned}
K_{p} & =1 / k_{p} !\left(k_{p}+\left|r_{p}\right|\right) !, \quad p=1,2,3, \cdots, N, \\
q & =2\left(\beta_{N}-k_{N}\right) .
\end{aligned}
$$

With mathematical induction, we find that $I\left(n ; r_{1}, r_{2}, \cdots, r_{N} ; \alpha\right)$ satisfies the recursion relationship

$$
\sum_{k=0}^{N} \sum_{M_{k}=-1}^{1} C\left(M_{k}, N\right) I\left(t_{k} ; S_{1, k}, S_{2, k}, \cdots, S_{N, k} ; \alpha\right)=0
$$

where

$$
\begin{aligned}
C\left(M_{k}, N\right) & =\left\{\begin{array}{ll}
\frac{2 \alpha}{N+1} & \text { if } M_{k}=0 \\
-1 & \text { if } M_{k}=-1,1
\end{array}\right\}, \\
t_{k} & =n+1+M_{0} \delta_{k, 0}, \\
S_{a, k} & =r_{a}+1+M_{a} \delta_{k, a}, \\
\delta_{k, a} & =\left\{\begin{array}{ll}
1 & \text { if } k=a \\
0 & \text { if } k \neq a
\end{array}\right\},
\end{aligned}
$$

and $I\left(t_{k} ; S_{1, k}, S_{2, k}, \cdots, S_{N, k} ; \alpha\right)$ represents either $T\left(t_{k} ; S_{1, k}, S_{2, k}, \cdots, S_{N, k} ; \alpha\right)$ or $U\left(t_{k} ; S_{1, k}, S_{2, k}, \cdots, S_{N, k} ; \alpha\right)$.

IV. Special Two-Dimensional Polynomials. Sometimes, recursion relationships arise which are similar to Eq. (2.5) but differing in the coefficients of the $r$ 's. 
Consider the recursion relationship

$$
2 \alpha I(n+1 ; r+1 ; \beta, \gamma ; \alpha)-\beta I(n+2 ; r+1 ; \beta, \gamma ; \alpha)
$$

$$
\begin{aligned}
-\beta I(n ; r+1 ; \beta, \gamma ; \alpha)- & \gamma I(n+1 ; \gamma+1 ; \beta, \gamma ; \alpha) \\
& -\gamma I(n+1 ; r ; \beta, \gamma ; \alpha)=0 .
\end{aligned}
$$

An extension of the Chebyshev polynomials allows for the determination of the polynomials which satisfy Eq. (4.1).

Replacing $\alpha$ by

$$
\left[\frac{\alpha}{\beta}-\frac{\gamma}{2 \beta}\left(y+y^{-1}\right)\right]
$$

in the generating functions produces the polynomials

$$
\begin{aligned}
& T(n ; r ; \beta, \gamma ; \alpha)=\frac{n}{2} \sum_{m=0}^{[n / 2]} \frac{(-)^{m+r}(n-m-1) !}{m !}\left(\frac{\gamma}{\beta}\right)^{n-2 m} \sum_{k=0}^{[\beta]} \frac{K(2 \alpha / \gamma)^{q} H(q)}{q !}, \\
& U(n ; r ; \beta, \gamma ; \alpha)=\sum_{m=0}^{[n / 2]} \frac{(-)^{m+r}(n-m) !}{m !}\left(\frac{\gamma}{\beta}\right)^{n-2 m} \sum_{k=0}^{(\beta)} \frac{K(2 \alpha / \gamma)^{q} H(q)}{q !},
\end{aligned}
$$

where $\beta, K, q$, and $H$ are the same as for Eq. (2.4).

The $T$ and $U$ polynomials of Eqs. (4.2) and (4.3) satisfy Eq. (4.1).

V. Comments. A solution to Eq. (1.5), where $I$ does not necessarily represent $T$ or $U$, can be written in terms of the Chebyshev polynomials. It appears that solutions to the higher-order recursion relationships should consist of combinations of the extended Chebyshev polynomials.

Acknowledgments. I am indebted to Professor Earl T. Kinzer for his helpful discussions.

Division of Natural Science and Mathematics

Floyd Junior College

Rome, Georgia 30161

1. G. Arfren, Mathematical Methods for Physicists, 2nd ed., Chap. 13, Academic Press, New York, 1970

2. S. Katsura \& S. INawashiro, "Lattice Green's functions for the rectangular and the square lattices at arbitrary points," J. Math. Phys., v. 12, 1971, pp. 1622-1630. MR 44 \#1367.

3. H. Margenau \& G. Murphy, The Mathematics of Physics and Chemistry, 2nd ed., Van Nostrand, Princeton, N.J., 1956. MR 17, 558. 